Conclusions: FAD is a diagnostic test that has the potential to accurately diagnose diskogenic LBP without the inherently painful need of a PLD.

\section{Poster 216 \\ Utility of Ultrasound in Evaluation of a Spontaneous Plantar Fascia Rupture: A Case Report.}

Michael J. Louwers, MD (University of Michigan, Ann Arbor, MI); Percival Pangilinan, MD; Brian Sabb, DO.

Disclosures: M. J. Louwers, None.

Patients or Programs: A 37-year-old man with no history of foot pain, presenting with a spontaneous plantar fascia rupture.

Program Description: The patient presented after experiencing medial left foot pain during a basketball game. The patient was abruptly changing directions when he felt a popping sensation and immediate, intense pain in the medial aspect of his left heel. The patient was able to walk with moderate discomfort but noticed bruising and swelling about 2 hours after the injury. There was no history of plantar fasciitis or any lower limb steroid injections. Examination revealed mild pes planus and hyperpronation of the midfoot bilaterally, with a small area of ecchymosis over the calcaneal origin of the left plantar fascia. Ultrasound of the left ankle and foot were performed and revealed severe fasciitis at the calcaneal insertion with partial tearing.

Setting: Tertiary care hospital.

Results: An Aircast boot was prescribed for 3 weeks for the left foot, as well as ibuprofen for pain relief. Afterward, we recommended minimal weight-bearing exercises for 2 additional weeks. At 5 weeks post injury, the patient was able to return to full activities without discomfort.

Discussion: Plantar fascia tears are usually associated with pre-existing plantar fasciitis or previous steroid injections. Spontaneous rupture, however, has rarely been reported in the literature. Similar to the other reports of spontaneous rupture, our patient had no previous symptoms or steroid injections, but did have risk factors including pes planus and hyperpronation. Ultrasound offers unique benefits over MRI in evaluation of this pathology. Ultrasound is ideal for superficial structures such as the plantar fascia; it is reliable, compact and inexpensive. Moreover, it is fast, noninvasive, provides the option of real-time imaging for dynamic testing and is an excellent alternative for claustrophobic patients.

Conclusions: Although rare, spontaneous rupture of the plantar fascia can occur during acceleration injuries such as running or jumping. In these patients, ultrasound provides a quick, accurate, and cost-effective means of evaluating plantar fascia tears.

\section{OTHER REHABILITATION TOPICS}

\section{Poster 217}

\section{A Brief History of Post-Polio in the United States.}

Lauro Halstead, MD (National Rehabilitation Hospital, Washington, DC); Judith A. Glaser, DO.

Disclosures: L. Halstead, None.

Objective: To describe the history of the late effects of polio in this country from 1980 to the present.

Design: Using the keywords post-polio, post-polio syndrome, late effects of polio, post-polio sequelae and postpoliomyelitis, we reviewed the number of articles in English listed in PubMed at 5-year intervals between 1980-2009. In addition, we compiled data from Post-Polio Health International records on the number of post-polio support groups and polio-dedicated clinics at 5-year intervals from 19852010

Main Outcome Measures: Historical assessment Results: In 1980, there was 1 article dealing with post-polio issues. This number increased dramatically during the 1980s and early ' 90 s peaking in 1995 when 64 were published. This figure declined over the next 14 years to 18 in 2009. Support groups and clinics showed a similar pattern of rise and decline with a maximum of 298 support groups and 96 clinics in 1990. These numbers declined to 131 and 32, respectively, by 2010. These data reflect a well-defined history of post-polio in this country that spanned approximately 30 years. During the 1980s and early 90s, there was a period of optimism that energized research, clinical and self-help initiatives. As the limits of these efforts became apparent during the late ' 90 s and early 2000s, resources and activities declined resulting in frustration and disillusionment within the post-polio community.

Conclusions: The late effects of polio were an unexpected sequelae of the mid-century polio epidemics in this country that resulted in a rise and fall of polio-related activities confined to a span of 3 decades. Nevertheless, there are still many thousands of polio survivors who will continue to need physiatric services for many years to come.

\section{Poster 218}

A Randomized, Double-blind, Placebocontrolled Crossover Study Using a LowFrequency Magnetic Field in the Treatment of Fatigue in Multiple Sclerosis.

Giampaolo Brichetto, MD, PhD (Italian Multiple Sclerosis Society, Genova, Italy); Mario Alberto Battaglia; Giovanna Konrad; Maria Laura Lopes de Carvalho, MD; Roberta Motta.

Disclosures: G. Brichetto, None.

Objective: One of the symptoms causing the greatest morbidity and disability in multiple sclerosis (MS) is fatigue. The aim of this randomized, double-blind, placebo-controlled 